\title{
Improving Men's Underwear Design by 3D Body Scanning Technology
}

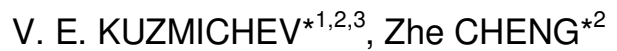 \\ ${ }^{1}$ Textile Institute, Ivanovo State Polytechnic University, Ivanovo, Russian Federation; \\ ${ }^{2}$ Institute of Clothing, Wuhan Textile University, Wuhan, Hubei, P. R. China; \\ ${ }^{3}$ Vladivostok State University of Economics and Service, Vladivostok, Russian Federation
}

DOI: 10.15221/16.016 http://dx.doi.org/10.15221/16.016

\begin{abstract}
This research devoted to the scanning technology of improving an underwear design by means of studying male bodies morphology. Increasing demands of contemporary men about an underwear have affected the way of designing including the choosing of structure, construction, knitted materials in accordance with the body morphology, perception, possible body reshaping, etc. It requires a combination of traditional design approaches with new data base. Traditional approach used limited number of body sizes and some empirical values, but this simple data base is not fully to reflect the male bodies morphology if the fit, comfort and additional functionalities of products should be reduced. Our exploration takes into attention the opinions and demands of more than 300 young people (Chinese, Russian, Indian). The underwear they have bought most appeared the size and front crotch discomfort; moreover, the majority of respondents said, simple way of underwear sizing (such as $S, M$, $\mathrm{L}, \mathrm{XL}, \mathrm{XXL}$...) doesn't give them good choice during buying underwear. So, the traditional basic body sizes for underwear design are outdated and unreasonable, it should be readjusted and reformed. We used the body scaner Vitus Smart XXL to create new data base for underwear design.
\end{abstract}

Keywords: 3D body scanning, body dimensions, men's underwear, morphology

\section{Introduction}

Contemporary styles, new materials and functional design of men's underwear is gradually improved, it has become one of the fastest growing garment industry. While men's underwear product improvement, design optimization can be obtained not only through style and pattern structure, but also needs to update new human body data including new body dimensions for using in underwear pattern making [1]. Cotemporary data base for men's underwear design is currently unable to meet and satisfy the growing demands of comfort and additional functionalities. As we know, the results of anthropometric measurements will vary depending on the situation and have a huge impact by main factors such as measured posture, breathing and measurement techniques used. By means of Anthroscan we can copy a real body in a steady posture when it is scanned.

Anthroscan is a software for the visualization, processing and evaluation of 3D scan data in general delivered by the Vitus Smart XXL 3D body scanner. Anthroscan is using in place of the anthropometric measurements as practical tool. This tool allows to observe the measurement of body (see fig. 1a). Each dimensions of body has a separate 4 digit code / number, such as hip girth 7525 (fig. 1b).

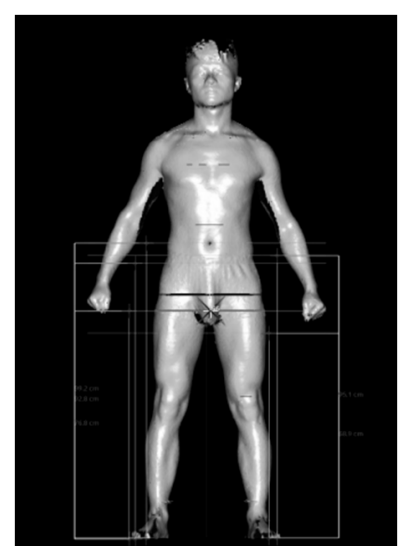

(a)

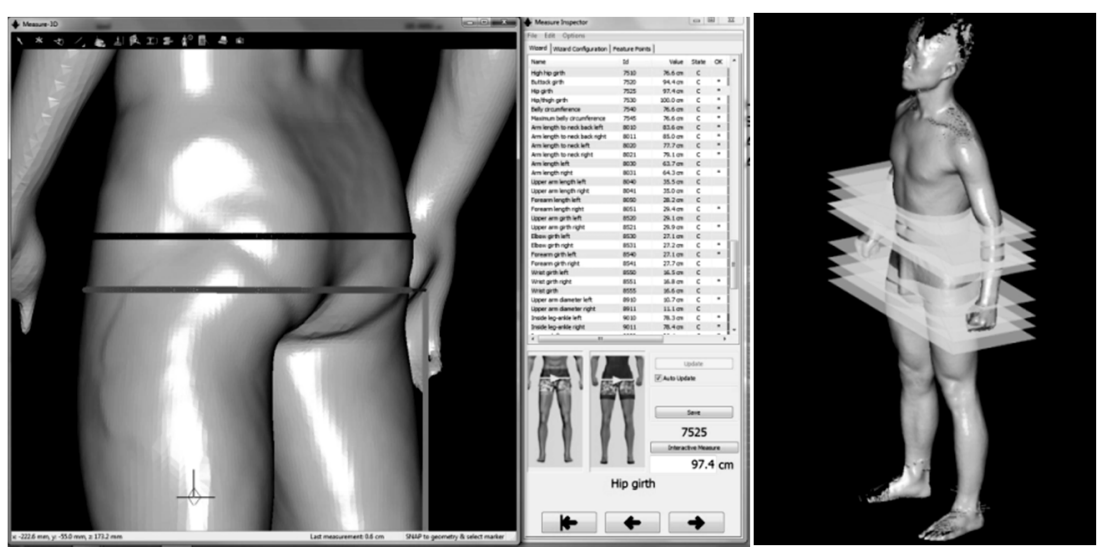

(b)

Fig. 1. Scheme of measuring of traditional (a) and new body dimensions (b)

*E-mail: wkd37@list.ru, zcheng@wtu.edu.cn 
In traditional methods of pattern making, most data used comes from the direct dimensions related to finished underwear such as waist girth, full crotch length, and others. In some of the existing design methods, most using natural waist girth (6510), hip girth (7520) and the full length of the crotch (6010), which is obviously impossible to complete to describe the different body characteristics. The traditional dimensions can not describe all features of male low torso in detailed. This schedule doesn't includes some crucial dimensions of human body to be sure about fit and comfort especially for daily, sports, shaping underwear. So, few basic dimensions of human body that applied to its structural design only used now. Therefore, it is necessary to combine the existing 3D body scanning technology to get additional body sizes to underwear design.

So, data base of body morphology should to be increased for the underwear design.

By early experiments, we have studied on men's underwear comfort [2, 3] and structural design $[4,5]$ for a more comprehensive and integrated analyzed. We have obtained some basic theory about men's underwear and crucial dimensions data which needed to provide a basis for subsequent experiment.

\section{Method of exploration}

Measurement of 38 males aged 20 to 26 year have been done by 3D laser scanner body VITUS Smart $X X L$ (4 columns) [6]. Average body sizes are height $174 \pm 5.5 \mathrm{~cm}$, natural waist girth $75.5 \pm 5.2 \mathrm{~cm}$, hip girth $98 \pm 4.8 \mathrm{~cm}$. Standard measurement position was in accordance with ISO 7250 [7], the average circumference error $<1 \mathrm{~mm}$, and the measured body wearing their underwear.

The body dimensions were measured by two ways: firstly, directly by 3D body scanner Vitus Smart $X X L$, secondly, after analyzing the horizontal and vertical cross-sections taken from image processing by software Anthroscan.

Through the measuring of male bodies by 3D body scanner, we created the schedule of new body characteristics of lower part. We have selected and analyzed many crucial measurements after 3D body scanning (more than 20 ) to detailed describing the male body morphology and can be applied to a new mapping method (see fig. 2) [8].

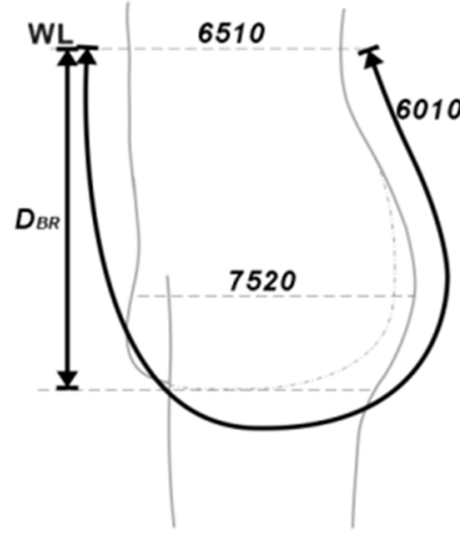

Traditional

(a)

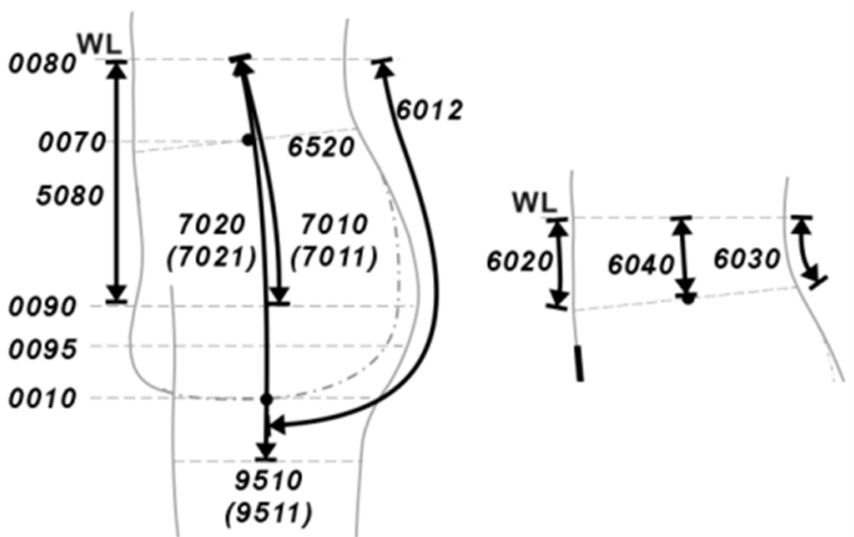

New

(b)

Fig. 2. Scheme of measuring of traditional (a) and new body dimensions (b):

(a) 6510-nature waist girth (the narrowest girth at waist level), DBR-nature waist level to crotch level (vertical distance), 6010-full crotch length (from nature waist front to back), 7520—hip girth (largest girth);

(b) 0080 - nature waist height (to floor), 0070 - waistband height (side point height), 5080—nature waist level to hip level (vertical distance), 0090 - hip height, 0095-low hip height (front genitalia peak point height, follow the wearing habits), 0100—crotch level height, 6020 (front), 6040 (side), 6030 (back)—nature waist level to waistband (according to the body skin), 6520 - waistband girth, 7020 (left), 7021 (right)—nature waist level to thigh level (according to the body skin), 7010 (left), 7011 (right)—nature waist level to thigh level (largest girth), 0997-nature waist level to crotch level (vertical distance), 9510 (left), 9511 (right)—thigh girth (largest girth), 6012-crotch length back

As we can see on Fig.2,new body dimensions can describe more exactly the features of male morphology including the position of belt, position of more prominent points, etc. 


\section{Results and discussion}

\subsection{New body sizes}

We have collected the profile silhouettes of European and Asian males to find new additional body dimensions which we are going to use in underwear design:

1) torso girth below natural waist in $1 \mathrm{~cm}$ to $20 \mathrm{~cm}$;

2) thigh girth in sloping direction from $0^{\circ}$ to $60^{\circ}$;

3) different outlines; the proportion between front and back parts of hip girth; the range of proportion from $46 \%$ to $54 \%$;

4) height of front and back soft tissue.

Fig. 3 a shows the underwear belt design area.

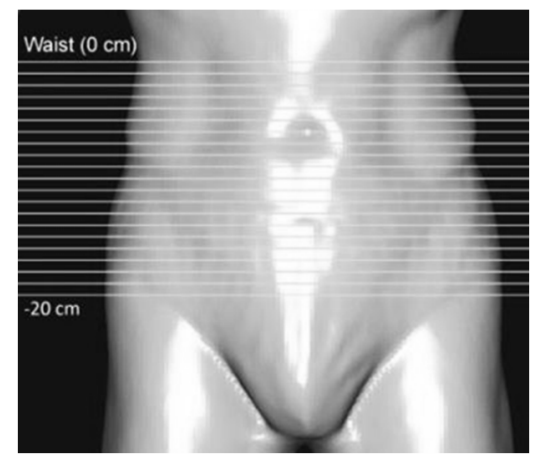

(a)

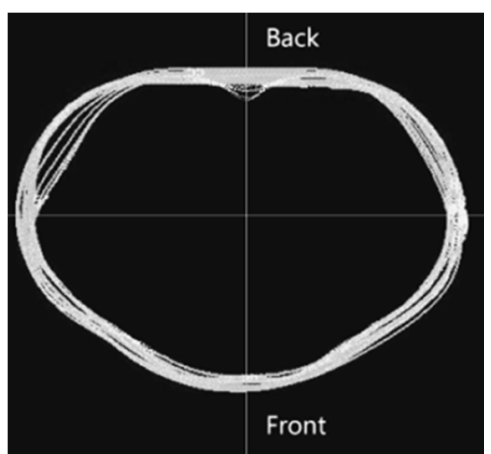

(b)

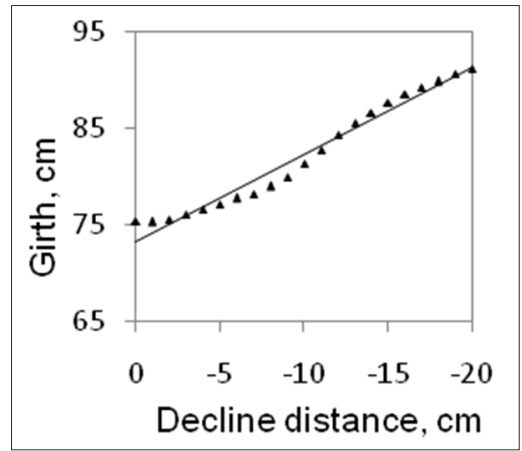

(c)

Fig. 3. Cross-sections of male torso taken between waist and lowest possible position of waistband (a), cross-sections of torso joined (b) and diagram for calculation of waistband girth (c)

The position of waistband will directly affect its size, although the belt material has good flexibility, but the belt can not only be based on natural waist girth. Therefore, we need to analyze the dimensions of this area at different heights.

Because the waistband of underwear located below natural waist, we have tried to find the torso girth below natural waist. Through our exploration of male bodies, the average distance between nature waist level to hip level (5080) is $23.9 \pm 1.2 \mathrm{~cm}$, so we have chosen the maximum distance from the nature waistline to possible waistband position as $-20 \mathrm{~cm}$ and declined it per $1 \mathrm{~cm}$ in experiment. Value $-20 \mathrm{~cm}$ means lowest waistband position in male underwear.

We have got the horizontal cross-sections of torso below waist in 1 to $20 \mathrm{~cm}$ (see fig. 4a). We named this distance that measured between natural narrowest waist and waistband location as " $h 1$ ". From fig. $3 \mathrm{~b}$ we can see how the configuration of torso girth is changing. By our tests, the average nature waist (0080) girth was $75.5 \pm 5.2 \mathrm{~cm}$, in $9 \mathrm{~cm}$ below nature waistline is the waistband line (6520) (average height of the measured underwear located about $4 \mathrm{~cm}$ below the navel), the average waistband girth was $79.9 \pm 4.8 \mathrm{~cm}$. In the tests of underwear samples an underwear waistband girth was significantly greater than natural waist girth, so the calculation of waistband size must be more precise.

Based on our testes, we found the equation for calculating the torso girth $G_{N w}$ as 1 st new additional body dimension:

$$
G_{N W}=72.63-1.01 h_{1}, \quad r=0.9899
$$

where: $G_{N W}$ is new torso girth below waist, $\mathrm{cm}$; $h 1$ is decline distance, $\mathrm{cm}, r$ is correlation coefficient. Underwear for body shaping can change buttock girth and shape, thigh volume, it will directly affect the underwear style and wearing comfort. Fig. 4 shows the position of point $a$ (on hip level) and point $b$ on underwear bottom that depends from underwear bottom types and girth.

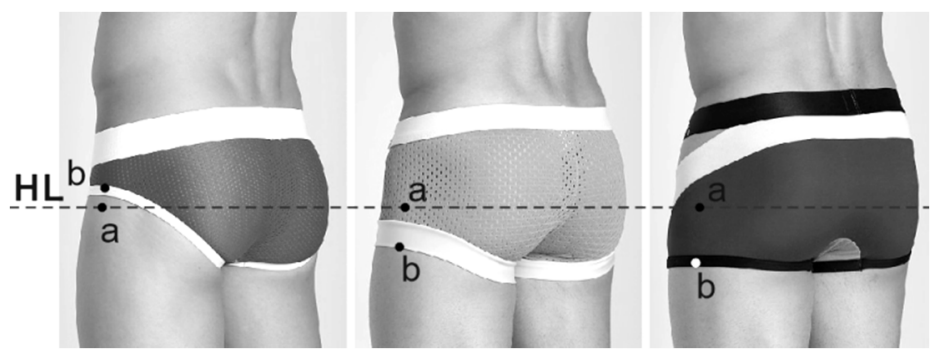

Fig. 4. Possible positions of underwear bottom 
To calculate the length of underwear bottom, we need know not only tight girth in horizontal direction but in sloping also. Fig. 5 shows how after scanning we have cut the thigh by several cross-sections per $0^{\circ}$ to $60^{\circ}$.

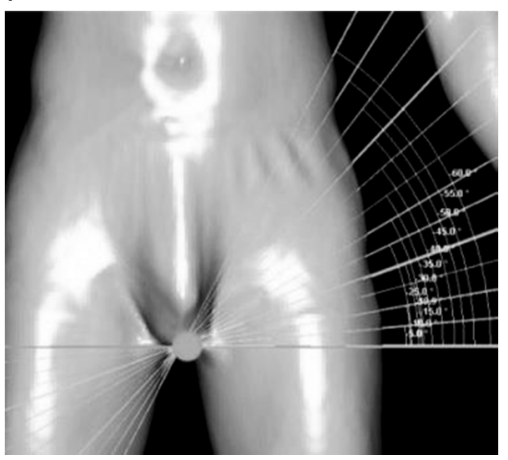

(a)

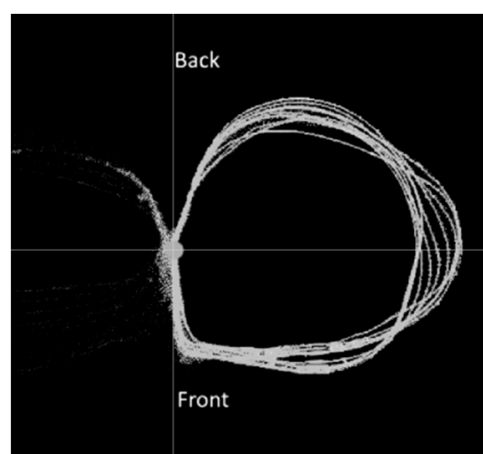

(b)

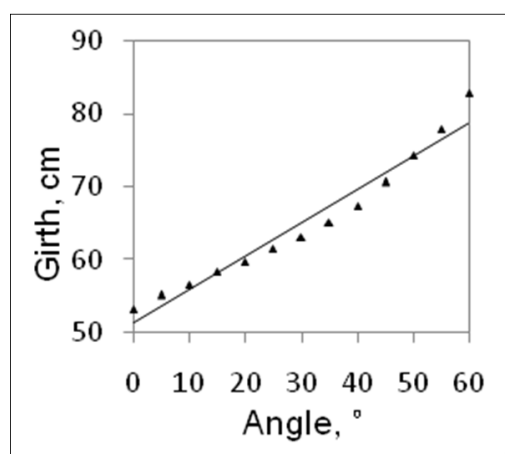

(c)

Fig. 5. Angles of thigh cutting (a), cross-sections of thigh (b) and diagram for tight sloping girth calculation

This range of angles reflects the possible positions of bottom hem of underwear. By our tests, the average thigh girth $(9510 / 9511)$ is $52.9 \pm 5.1 \mathrm{~cm}\left(0^{\circ}\right)$ and $83.1 \pm 4.7 \mathrm{~cm}\left(60^{\circ}\right)$. Based on our tests, we have found the equation for calculating new tight sloping girth $G_{T N}$ (as the bottom girth for underwear) as 2nd new additional body dimension:

$$
G_{T N}=0.48 x+51.31, \quad r=0.9772
$$

where: $G_{T N}$ is tight scoping girth, $\mathrm{cm} ; x$ is angle, ${ }^{\circ} ; r$ is correlation coefficient.

Fig. 6 a shows the way of measurements cross-sections of low torso. The proportion between front and back parts of hip girth has changed from $46 \%$ to $54 \%$ (see fig. 6b). The vertical cross-sections of low parts of torso were obtained to find the typical curved lines (see fig. 6c).

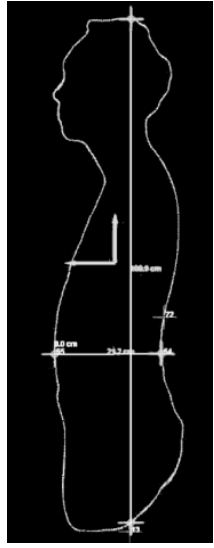

(a)

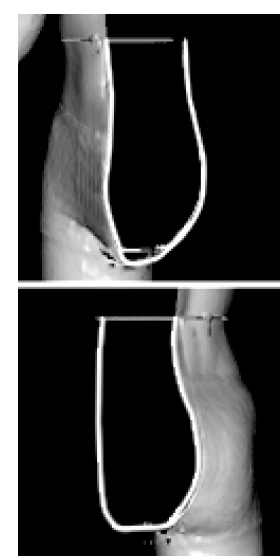

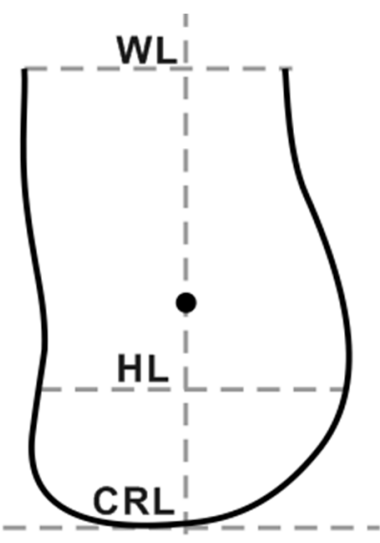

(c)

Fig. 6. Torso vertical cross-sections (a), low parts of torso joined vertical cross-sections (b), average vertical cross-sections draw for males with next sizes $B G=95.5 \mathrm{~cm}, W G=75.5 \mathrm{~cm}, \quad H G=97.3 \mathrm{~cm}$

As fig. 6c shown, the lower part of torso has different configurations, although the hip deviation of measured bodies is small. It will influence on underwear styles, way of wearing and different human physiological structure characteristics and other. All factors will affect the profile silhouettes on the abdomen parts, genitals, and buttocks.

\subsection{Experiments of correction effects}

Main effects of body correction (shaping) by means of men's underwear (push-up effect) shown in fig. 7a. Outline shapes shown by dotted line before shaping and solid line after wearing corrective underwear which lifted the genitals and buttocks (soft tissue). As usual, different styles of underwear produced different lift [8]. Set of 12 samples were measured in Anthroscan software to find the center of the front genitals. Because of the experimental factors (must nudity measure), therefore the small number of the test samples. 
As fig. 7 shown the correction effects (push-up effect) in front and rear after dressing the underwear (for daily life underwear this effect mostly exists in front). By our tests, the push-up distance in front is from 2.1 to $8.8 \mathrm{~cm}$, in back is from 0.2 to $1.1 \mathrm{~cm}$.

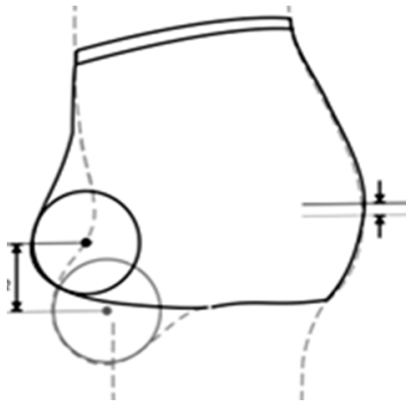

a

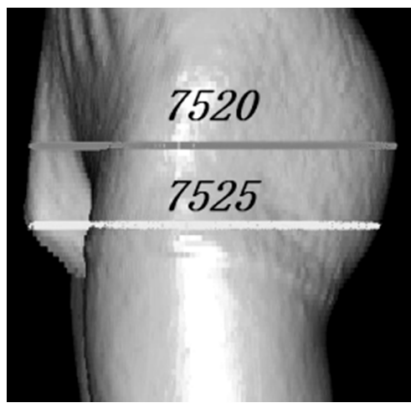

$b$

Fig. 7. Push-up effect in front and rear

\section{Conclusion and future research}

Using new list with body sizes, we found the main differences between the Russians and the Chinese, such as,

1) relationships existing between heights and girths on waist and bottom lines;

2) shapes of sections on hip (the width of hip peak) and lower hip (the width of genitals peak);

3) configurations of waist, hip and thigh cross-sections;

4) different effects of "push-up" (lifting / correct the soft tissues of body) at front and rear.

We can calculate push-up effect after the scanning nude bodies, on one side, and the dressing bodies for different types of underwear, on the other side.

We prepared the recommendations how to design the underwear pattern block for the Russians and the Chinese, to choose the structure of underwear with more reasonable and satisfy characteristics in accordance with the male body morphology. Furthermore, the new data base could help to create more detailed classification of male underwear with particular and additional sizes to make the consumer choices easier and clearer.

This research provides detailed and clear approach to describe men's underwear and male bodies' morphology, new way to advance mass production and individual customization with body scanning technology. It will be possible to connect the features of body, material and structure design into one system.

\section{References:}

[1] C.K.AU, Y.S.MA. "Garment pattern definition, development and application with associative feature approach", in Computers in Industry, Vol.61, No.6, 2010, pp.524-531. http://dx.doi.org/10.1016/j.compind.2010.03.002

[2] Zhe CHENG, V.E.KUZMICHEV, Yue LI, et al. "Evaluation Analysis on Pressure and Tensile Property of Knitted Materials for Men's Underwear", in Journal of Wuhan Textile University, Vol.6, No.27, 2014, pp.32-35. http://doi.org/10/f3rgh9

[3] Zhe CHENG, V.E.KUZMICHEV, D.C. ADOLPH. "Choosing of knit materials for men's compression", in Sewing Industry, Vol.5, 2014, pp.25-29.

[4] Zhe CHENG, V.E.KUZMICHEV, Yue LI. "Test and Analysis on Properties of Men's Underwear Fabrics", in Advanced Textile Technology, Vol.22, No.6, 2014, pp.48-50.

[5] Zhe CHENG, V.E.KUZMICHEV, Yue LI. "Analysis of Male Lower Part of Body and Underwear Structure", in Journal of Wuhan Textile University, Vol.3, 2015, pp.49-52. http://doi.org/10/f3qidj

[6] Anthroscan User Guide Version 3.x. Kaiserslautern: Human Solutions GmbH, 2009, 6:65-70.

[7] Basic human body measurements for technological design - Part 2: Statistical summaries of body measurements from individual ISO populations (ISO/TR 7250-2:2010); German version CEN ISO/TR 7250-2: 2011,7.

[8] Zhe CHENG, V.E.KUZMICHEV. "Methods of designing men's compression underwear", in Sewing Industry, Vol.2 2015, pp.45-49.

[9] Zhe CHENG, V.E.KUZMICHEV. "Art and structural data base for the design of men's underwear", In Sewing Industry, Vol.6, 2013, pp.26-29. 